Research, Society and Development, v. 9, n. 3, e181932700, 2020

(CC BY 4.0) | ISSN 2525-3409 | DOI: http://dx.doi.org/10.33448/rsd-v9i3.2700

\title{
Segurança do paciente no âmbito domiciliar: uma revisão integrativa da literatura
}

Patient safety at home: an integrative literature review

Seguridad del paciente en el hogar: una revisión bibliográfica integradora

Recebido: 30/01/2020 | Revisado: 05/02/2020 | Aceito: 21/02/2020 | Publicado: 09/03/2020

Andressa da Silva Alves

ORCID: https://orcid.org/0000-0002-4844-0035

Universidade Paulista, Brasil

E-mail: andressa321silva@gmail.com

Ricardo Saraiva Aguiar

ORCID: https://orcid.org/0000-0003-0335-2194

Universidade Paulista, Brasil

E-mail: ricardo.aguiar@docente.unip.br

\section{Resumo}

Analisar o desenvolvimento de ações voltadas para a assistência à saúde no ambiente domiciliar com enfoque na segurança do paciente. Trata-se de uma revisão integrativa da literatura realizada a partir das bases de dados SCIELO, LILACS e BDENF. Refinou-se a busca de publicações para o período de 2005 a 2018, sendo realizada uma análise crítica dos estudos encontrados por meio da síntese narrativa. Para a elaboração da pergunta norteadora utilizou a estratégia PICO: como a assistência de enfermagem é organizada no ambiente domiciliar para promover um cuidado seguro? Foram selecionados 16 artigos para interpretação, discussão e composição deste estudo. Assim, foram definidas duas categorias para a discussão dos resultados: prevenção de riscos e danos nos cuidados prestados no âmbito domiciliar; e a enfermagem e a segurança do paciente no ambiente domiciliar. $\mathrm{O}$ estudo demonstrou que os profissionais de enfermagem possuem papel fundamental no estabelecimento da segurança do paciente no âmbito domiciliar, na medida em que se torna responsável pela realização das principais intervenções necessárias durante o tratamento do indivíduo em seu domicílio.

Palavras-chave: Assistência Domiciliar; Enfermagem Domiciliar; Segurança do Paciente; Serviços de Assistência Domiciliar.

\section{Abstract}


To analyze the development of actions aimed at health care in the home environment with a focus on patient safety. This is an integrative literature review carried out from the SCIELO, LILACS and BDENF databases. The search for publications for the period from 2005 to 2018 was refined, with a critical analysis of the studies found through the narrative synthesis. The PICO strategy was used to elaborate the guiding question: how is nursing care organized in the home environment to promote safe care? 16 articles were selected for interpretation, discussion and composition of this study. Thus, two categories were defined for the discussion of results: prevention of risks and damage in care provided at home; and nursing and patient safety in the home environment. The study demonstrated that nursing professionals have a fundamental role in establishing patient safety at home, insofar as they become responsible for carrying out the main interventions necessary during the treatment of the individual at home.

Keywords: Home Nursing; Home Health Nursing; Patient Safety; Home Care Services.

\section{Resumen}

Analizar el desarrollo de acciones dirigidas a la atención de la salud en el hogar con un enfoque en la seguridad del paciente. Se trata de una revisión bibliográfica integradora realizada a partir de las bases de datos SCIELO, LILACS y BDENF. La búsqueda de publicaciones para el período comprendido entre 2005 y 2018 fue refinada, con un análisis crítico de los estudios encontrados a través de la síntesis narrativa. La estrategia PICO se utilizó para elaborar la pregunta orientadora: ¿cómo se organiza la atención de enfermería en el hogar para promover una atención segura? Se seleccionaron 16 artículos para la interpretación, discusión y composición de este estudio. Por lo tanto, se definieron dos categorías para la discusión de resultados: prevención de riesgos y daños en la atención brindada en el hogar; y enfermería y seguridad del paciente en el entorno del hogar. El estudio demostró que los profesionales de enfermería tienen un papel fundamental en el establecimiento de la seguridad del paciente en el hogar, en la medida en que se hacen responsables de llevar a cabo las principales intervenciones necesarias durante el tratamiento del individuo en el hogar.

Palabras clave: Atención Domiciliaria de Salud; Cuidados de Enfermería em el Hogar; Seguridad del Paciente; Servicios de Atención de Salud a Domicilio.

\section{Introdução}


A segurança do paciente é um dos fundamentos essenciais na assistência à saúde e um elemento indispensável para gestão da qualidade em âmbito mundial (Tase, Quadrado \& Tronchin, 2018). Essa tem sido um assunto de debate entre gestores e equipes de saúde a nível global e está mais presente nos roteiros das reuniões de instituições de saúde públicas e privadas após a publicação do relatório americano "Errar é humano: construindo um sistema de saúde mais seguro" do Instituto de Medicina (IOM) (Golle, Ciotti, Herr, Aozone, Schmidt \& Kolankiewicz, 2018; Lemos, Azevedo, Bernardes, Ribeiro, Menezes, \& Mata, 2018). O documento aponta a ocorrência de iatrogenias em um pouco mais de $3 \%$ do total de prontuários analisados. Além disso, os autores referiram o óbito de 44.000 a 98.000 pacientes por ano nos Estados Unidos da América (EUA), cujo as causas foram incidentes, que poderiam, a maior parte, ser evitados (Institute of Medicine, 1999).

Diante disso, a assistência domiciliar deve ser compreendida como um conjunto de atividades e ações de prevenção, ensino, recuperação e manutenção da qualidade de vida e saúde, realizado em prol de pacientes e familiares em seu respectivo domicílio (Cunha, Santos, Lepinski, Santos, Hermann \& Lacerda, 2014).

Assim, a assistência de enfermagem no âmbito domiciliar pressupõe a prática profissional com aspectos não controláveis, pois o ambiente é controlado pela família e não pelo profissional de saúde. Dessa forma, o gerenciamento da assistência prestada em domicílio acaba sendo pautado por um alto grau de complexidade, tendo em vista que a atuação da equipe de saúde ocorre em um ambiente sensível, exigindo do profissional de saúde a permissão para atuação no domicílio (Dalben, 2011).

$\mathrm{Na}$ contemporaneidade, é notável o aumento da preocupação em relação à área da segurança do paciente, tendo em vista a necessidade de reduzir os potenciais danos e riscos desnecessários associados à assistência em saúde a um patamar mínimo aceitável. (Dalben, 2011).

Ademais, em virtude dos contínuos casos de erros no processo de assistência a saúde, nota-se a necessidade de implementação de protocolos específicos capazes de subsidiar a tarefa de cuidado e segurança na respectiva área, visando à redução do número de ocorrências e de eventuais eventos adversos, ou seja, incidentes que podem acontecer durante a prestação de cuidados à saúde e que podem ocasionar em prejuízo e dano ao paciente (World Health Organization, 2009; Brasil, 2013).

Dessa forma, o presente estudo se justifica pela busca da compreensão acerca da importância do estabelecimento de protocolos de segurança do paciente durante a realização de tratamentos no ambiente domiciliar, assim como quais devem ser os cuidados, métodos e 
protocolos a serem seguidos visando a garantia de maior qualidade na oferta de cuidados e consequentemente mais segurança ao paciente durante a realização de cuidados em seu ambiente domiciliar.

Portanto, esta pesquisa objetiva analisar o desenvolvimento de ações voltadas para a assistência à saúde no ambiente domiciliar com enfoque na segurança do paciente. Sob essa perspectiva, destaca-se a questão que norteou a presente proposta investigativa: como a assistência de enfermagem é organizada no ambiente domiciliar para promover um cuidado seguro?

\section{Metodologia}

Trata-se de uma revisão integrativa da literatura, uma vez que contribui no processamento sistemático e analítico dos resultados, pois é característica da busca de informações sobre um assunto ou tema que resuma a situação da ciência sobre um problema de pesquisa, visando a clareza do determinado tema (Aguiar, Santana \& Santana, 2015).

Para a elaboração da pergunta norteadora utilizou a estratégia PICO: P - população e problema; I - intervenção; C - comparação; e $\mathrm{O}$ - outcome (termo em inglês que significa desfecho $)^{7}$. Assim, considerou-se P: pacientes domiciliares; I: cuidado seguro; C: qualquer comparação relacionada a assistência à saúde; O: segurança do paciente. Nesta direção, a pergunta construída foi: como a assistência de enfermagem é organizada no ambiente domiciliar para promover um cuidado seguro?

Tal pesquisa foi estruturada em três etapas, a saber: identificaram-se os descritores junto à Biblioteca Virtual em Saúde (BVS) através dos descritores em ciências da saúde (DeCS), selecionando aqueles considerados pertinentes para a consecução da pesquisa. $\mathrm{Na}$ segunda etapa, realizou-se a pesquisa por meio dos descritores nas bases de dados Scientific Eletronic Library Online (SCIELO), Literatura Latino-Americana e do Caribe em Ciências da Saúde (LILACS) e Base de Dados em Enfermagem (BDENF) utilizando o operador booleano and entre eles (segurança do paciente and enfermagem domiciliar and serviços de assistência domiciliar and assistência domiciliar), refinando a busca para o período de 2005 a 2018; e por fim, procedeu-se com a análise crítica dos estudos, excluindo aqueles não condizentes com o escopo da pesquisa, bem como as produções duplicadas.

Nessa perspectiva, a análise dos estudos encontrados foi sistematizada seguindo as etapas da pesquisa bibliográfica, contemplando: o levantamento bibliográfico preliminar nas bases de dados supracitadas; a viabilidade dos estudos encontrados para a revisão literária; a 
leitura seletiva, analisando, de maneira específica, a pertinência dos estudos; a leitura analítica, sumarizando as informações encontradas de maneira crítica; a leitura interpretativa, articulando os conhecimento versados em todos os estudos analisados; e a elaboração do texto final que sintetiza os resultados da pesquisa literária (Mendes, Silveira \& Galvão, 2019).

Em suma, como critério de inclusão dos estudos, selecionou-se as produções científicas brasileiras, concretizadas entre os anos de 2005 a 2018, que versavam sobre o objeto do estudo. Como critérios de exclusão, enquadraram-se artigos disponíveis em bases de dados internacionais e exclusivamente em língua estrangeira.

Com base nos critérios de inclusão, foram selecionados 16 artigos para interpretação, discussão e composição deste estudo utilizando a síntese narrativa para discussão dos dados.

\section{Resultados}

Foram utilizados 16 artigos nesta revisão integrativa, sendo interpretados e sintetizados todos os resultados, através de uma comparação dos dados evidenciados na análise dos artigos ao referencial teórico.

Quadro 1 - Distribuição dos artigos de acordo com o título, objetivo, conclusão e ano de publicação.

\begin{tabular}{|c|c|c|c|c|}
\hline $\begin{array}{l}\text { Ordem } \\
\text { dos } \\
\text { artigos }\end{array}$ & Título & Objetivo & Conclusão & Ano \\
\hline 1 & $\begin{array}{l}\text { Gerenciando a assistência } \\
\text { domiciliar }\end{array}$ & $\begin{array}{l}\text { Descrever como a assistência domiciliar é } \\
\text { vivenciada pelos gestores em saúde. }\end{array}$ & $\begin{array}{l}\text { Há necessidade de gestores em saúde } \\
\text { capacitados para oferecer um cuidado } \\
\text { sistematizado e de qualidade na } \\
\text { assistência domiciliar. }\end{array}$ & 2014 \\
\hline 2 & $\begin{array}{l}\text { Cuidados domiciliares: } \\
\text { interação do enfermeiro com a } \\
\text { pessoa idosa/família }\end{array}$ & $\begin{array}{l}\text { Compreender o processo de interação com a } \\
\text { pessoa idosa e família no domicílio } \\
\text { desenvolvido pelos enfermeiros. }\end{array}$ & $\begin{array}{l}\text { A categoria central foi "Construindo a } \\
\text { relação em contexto domiciliar" pelo fato da } \\
\text { relação da enfermeira com o idoso e a } \\
\text { família ser central em todo o processo de } \\
\text { cuidados. }\end{array}$ & 2012 \\
\hline 3 & $\begin{array}{l}\text { O enfermeiro frente aos riscos } \\
\text { ocupacionais em home care }\end{array}$ & $\begin{array}{l}\text { Identificar os riscos ocupacionais e descrever } \\
\text { ações e medidas preventivas para minimizar } \\
\text { riscos ao enfermeiro que atua em Home Care. }\end{array}$ & $\begin{array}{l}\text { Dentre as ações/medidas preventivas, } \\
\text { enfatiza-se a utilização de Equipamento de } \\
\text { Proteção Individual, orientação quanto ao } \\
\text { uso desses equipamentos e diminuição da } \\
\text { carga horária de trabalho. }\end{array}$ & 2011 \\
\hline 4 & $\begin{array}{l}\text { Assistência de enfermagem ao } \\
\text { paciente acamado em } \\
\text { domicílio: uma revisão } \\
\text { sistemática. }\end{array}$ & $\begin{array}{l}\text { Analisar a literatura existente acerca da } \\
\text { assistência de enfermagem ao paciente } \\
\text { acamado em domicílio. }\end{array}$ & $\begin{array}{c}\text { A atenção domiciliar à saúde tem se inserido } \\
\text { cada vez mais na organização do sistema de } \\
\text { saúde e o enfermeiro torna-se peça } \\
\text { fundamental nesse sentido. }\end{array}$ & 2015 \\
\hline 5 & $\begin{array}{l}\text { Qualidade dos cuidados } \\
\text { domiciliares em enfermagem a } \\
\text { idosos dependentes }\end{array}$ & $\begin{array}{c}\text { Contribuir para promover a compreensão dos } \\
\text { fatores envolvidos na qualidade dos cuidados } \\
\text { de enfermagem a idosos dependentes de } \\
\text { cuidados domiciliares. }\end{array}$ & $\begin{array}{l}\text { O principal obstáculo centra-se na escassez } \\
\text { de recursos e na desorganização, traduzidos } \\
\text { em falta de tempo, desordem e frustração. }\end{array}$ & 2013 \\
\hline 6 & $\begin{array}{l}\text { Atenção domiciliar na } \\
\text { estratégia saúde da família: } \\
\text { avaliação do grau de } \\
\text { implantação em Camaçari, } \\
\text { Bahia } \\
\end{array}$ & $\begin{array}{c}\text { Estimar o grau de implantação da Atenção } \\
\text { Domiciliar Tipo } 1 .\end{array}$ & $\begin{array}{l}\text { A atenção ao usuário foi o componente de } \\
\text { nível mais avançado, apesar da baixa } \\
\text { pontuação das equipes em relação à } \\
\text { promoção da saúde e à congestão do plano } \\
\text { de cuidados. } \\
\end{array}$ & 2017 \\
\hline 7 & $\begin{array}{l}\text { Assistência domiciliar em } \\
\text { saúde: subsídios para um }\end{array}$ & $\begin{array}{c}\text { Realizar uma análise comparada entre uma } \\
\text { proposta de assistência domiciliar no âmbito da }\end{array}$ & $\begin{array}{l}\text { A proposta analisada representa um passo } \\
\text { importante, todavia, a implementação deve }\end{array}$ & 2015 \\
\hline
\end{tabular}


Research, Society and Development, v. 9, n. 3, e181932700, 2020

(CC BY 4.0) | ISSN 2525-3409 | DOI: http://dx.doi.org/10.33448/rsd-v9i3.2700

\begin{tabular}{|c|c|c|c|c|}
\hline & $\begin{array}{l}\text { projeto de atenção básica } \\
\text { brasileira }\end{array}$ & $\begin{array}{l}\text { Atenção Básica no Brasil com o modelo } \\
\text { adotado pelo Canadá. }\end{array}$ & $\begin{array}{c}\text { levar em conta as limitações identificadas e } \\
\text { os desafios políticos e institucionais } \\
\text { existentes. }\end{array}$ & \\
\hline 8 & $\begin{array}{l}\text { Úlcera por pressão após a alta } \\
\text { hospitalar e o cuidado em } \\
\text { domicílio }\end{array}$ & $\begin{array}{l}\text { Identificar o perfil sociodemográfico e de } \\
\text { saúde de pacientes que necessitavam de } \\
\text { cuidados domiciliares após a alta. }\end{array}$ & $\begin{array}{c}\text { É necessário melhorar as orientações para o } \\
\text { cuidado domiciliar durante a hospitalização e } \\
\text { criar mecanismos de comunicação entre os } \\
\text { serviços de saúde. }\end{array}$ & 2016 \\
\hline 9 & $\begin{array}{l}\text { Sobre o cuidar e o ser cuidado } \\
\text { na atenção domiciliar }\end{array}$ & $\begin{array}{l}\text { Evidenciar experiências o cuidar e ser cuidado } \\
\text { e apresentar a visão de cuidadores familiares } \\
\text { sobre um serviço de atenção domiciliar. }\end{array}$ & $\begin{array}{l}\text { A alteridade vivida pelos cuidadores e a } \\
\text { sobrecarga de responsabilidades são algumas } \\
\text { expressões captadas no campo de pesquisa. }\end{array}$ & 2017 \\
\hline 10 & $\begin{array}{l}\text { Segurança do paciente no uso } \\
\text { de medicamentos após a alta } \\
\text { hospitalar: estudo exploratório }\end{array}$ & $\begin{array}{l}\text { Compreender a dinâmica e os desafios do } \\
\text { cuidado fornecido ao paciente pela equipe } \\
\text { hospitalar, visando à segurança no processo de } \\
\text { uso de medicamentos após a alta hospitalar. }\end{array}$ & $\begin{array}{l}\text { Conclui-se que são desenvolvidas algumas } \\
\text { estratégias, porém com limitações e sem } \\
\text { articulação adequada com outros serviços de } \\
\text { saúde para a continuidade do cuidado. }\end{array}$ & 2014 \\
\hline 11 & $\begin{array}{l}\text { Nutrição enteral domiciliar: } \\
\text { perfil dos usuários e cuidadores } \\
\text { e os incidentes relacionados às } \\
\text { sondas enterais }\end{array}$ & $\begin{array}{l}\text { Caracterizar o perfil de usuários e cuidados em } \\
\text { nutrição enteral domiciliar e analisar os } \\
\text { incidentes relacionados às sondas enterais. }\end{array}$ & $\begin{array}{c}\text { Ações como o monitoramento das } \\
\text { extubações e a implantação de medidas que } \\
\text { reduzam ou mitiguem tais eventos, podem } \\
\text { contribuir para assistência segura e livre de } \\
\text { danos. } \\
\end{array}$ & 2018 \\
\hline 12 & $\begin{array}{l}\text { Adesão ao regime terapêutico } \\
\text { medicamentoso e aspectos } \\
\text { biopsicossociais dos idosos } \\
\text { integrados em cuidados } \\
\text { continuados domiciliários }\end{array}$ & $\begin{array}{l}\text { Avaliar a associação entre os aspetos } \\
\text { biopsicossociais e a adesão ao regime } \\
\text { terapêutico medicamentoso dos idosos integra- } \\
\text { dos nas equipes de cuidados continuados } \\
\text { domiciliários. }\end{array}$ & $\begin{array}{l}\text { Conhecendo a multiplicidade de aspectos } \\
\text { envolvidos na adesão, sugere-se que os } \\
\text { mesmos sejam considerados nas } \\
\text { intervenções de enfermagem promotoras de } \\
\text { medidas de controle da terapêutica. }\end{array}$ & 2017 \\
\hline 13 & $\begin{array}{l}\text { Úlcera por pressão em } \\
\text { pacientes sob assistência } \\
\text { domiciliar. }\end{array}$ & $\begin{array}{l}\text { Investigar as características sociodemográficas } \\
\text { e clínicas e o risco para desenvolvimento de } \\
\text { úlcera por pressão em pacientes sob assistência } \\
\text { domiciliária. }\end{array}$ & $\begin{array}{l}\text { Identificou-se a necessidade de intervenção } \\
\text { educacional junto a esta população e aos } \\
\text { serviços de saúde. }\end{array}$ & 2010 \\
\hline 14 & $\begin{array}{l}\text { Correlação entre equilíbrio e } \\
\text { ambiente domiciliar como risco } \\
\text { de quedas em idosos com } \\
\text { acidente vascular encefálico }\end{array}$ & $\begin{array}{l}\text { Correlacionar o equilíbrio e o ambiente } \\
\text { domiciliar como risco de quedas em idosos } \\
\text { acometidos pelo acidente vascular encefálico. }\end{array}$ & $\begin{array}{l}\text { A dificuldade de equilíbrio apresentada pelos } \\
\text { idosos com acidente vascular encefálico } \\
\text { associada à alta prevalência de fatores de } \\
\text { risco encontrados no ambiente domiciliar } \\
\text { remete a um quadro preocupante, tornando- } \\
\text { se necessária a criação de medidas que visem } \\
\text { à prevenção das quedas. }\end{array}$ & 2010 \\
\hline 15 & $\begin{array}{l}\text { Cuidado seguro ao paciente: } \\
\text { contribuiçôes da enfermagem }\end{array}$ & $\begin{array}{l}\text { Refletir sobre a contribuição da enfermagem } \\
\text { para a segurança do paciente na construção do } \\
\text { cuidado seguro. }\end{array}$ & $\begin{array}{l}\text { É possível nortear de maneira mais segura as } \\
\text { ações realizadas pelos profissionais de } \\
\text { enfermagem visando o estabelecimento de } \\
\text { uma melhor assistência ao paciente sob seus } \\
\text { cuidados. }\end{array}$ & 2015 \\
\hline 16 & $\begin{array}{l}\text { Assistência de enfermagem e o } \\
\text { enfoque da segurança do } \\
\text { paciente no cenário brasileiro }\end{array}$ & $\begin{array}{l}\text { Analisar a contribuição da enfermagem para a } \\
\text { segurança do paciente no Brasil. }\end{array}$ & $\begin{array}{l}\text { As publicações demonstram o conjunto de } \\
\text { ações positivas aplicadas pela enfermagem } \\
\text { na segurança do paciente, refletindo a } \\
\text { relevância da atuação do profissional } \\
\text { enfermeiro. }\end{array}$ & 2016 \\
\hline
\end{tabular}

Fonte: Os autores (2018).

\section{Discussão}

Diante dos resultados, emergiram-se as seguintes categorias para discussão por meio de uma síntese narrativa: 1) Prevenção de riscos e danos nos cuidados prestados no âmbito domiciliar; 2) A enfermagem e a segurança do paciente no âmbito domiciliar.

\subsection{Prevenção de riscos e danos nos cuidados prestados no âmbito domiciliar}

A prestação de cuidados de enfermagem no âmbito domiciliar constitui um dispositivo que visa reorganizar o processo de trabalho dos profissionais da área de saúde, visando reduzir a demanda existente em virtude do atendimento no ambiente hospitalar ou do 
respectivo período de internação necessário ao tratamento de determinados casos. Além disso, a prática busca fortalecer a humanização do processo de atenção à saúde, intensificando a desinstitucionalização e ampliando a autonomia dos respectivos pacientes. Dessa forma, a prestação de tais cuidados baseia-se em um conjunto de atividades que visam garantir a promoção da saúde, prevenção e tratamento de doenças, assim como maior agilidade no processo de reabilitação do paciente por meio de ações desenvolvidas em domicílio (Rehem \& Trad, 2005; Gago \& Lopes, 2012; Cortez, Valente \& Ribeiro, 2011; Vieira, Bezerra, Sobreira, Silva \& Feitosa, 2015; Carvalhais \& Sousa, 2013; Lopes, Vilasbôas \& Castellanos, 2017).

Dessa forma, os cuidados de enfermagem prestados no ambiente domiciliar exigem uma série de precauções a serem adotadas pelo profissional visando a preservação de sua saúde e de seu paciente. Sendo assim, destaca-se a importância de compreensão dos riscos aos quais os indivíduos se expõem, tais como o contato com objetos perfurantes e cortantes, agentes biológicos diversos, elementos químicos e materiais de manipulação, permitindo que o profissional adote práticas preventivas que evitem quaisquer tipos de eventos adversos (Gago \& Lopes, 2012; Cortez, Valente \& Ribeiro, 2011; Vieira, Bezerra, Sobreira, Silva \& Feitosa, 2015).

Para evitar a incidência de danos à saúde do paciente, assim como o agravamento do seu quadro de saúde durante o tratamento no âmbito domiciliar, torna-se fundamental que as orientações referentes aos cuidados domiciliares que devem ser adotados pelo paciente sejam fornecidas pelo enfermeiro de maneira clara e precisa. Com isso, a enfermagem possibilita que tanto o paciente quanto o seu cuidador sejam capazes de evitar situações de conflito, por meio da promoção adequada de medicamentos necessários ao tratamento individual, bem como o desenvolvimento de precauções e estratégias capazes de promover a melhora do seu quadro de saúde e de sua qualidade de vida (Vieira, Bezerra, Sobreira, Silva \& Feitosa, 2015; Carvalhais \& Sousa, 2013; Lopes, Vilasbôas \& Castellanos, 2017; Monterroso, Sá \& Joaquim, 2017).

Dessa maneira, a oferta de cuidados de enfermagem no âmbito domiciliar é capaz de proporcionar o desenvolvimento de um novo ambiente de cuidado, configurando-se como um novo método de produção de projetos terapêuticos. Entretanto, destaca-se a importância do estabelecimento de um serviço pautado pela continuidade e integralidade do cuidado, visando garantir o atendimento da demanda dos pacientes e familiares, bem como a prestação de uma assistência à saúde pautada pelo respeito à dignidade humana e em busca do melhor tratamento (Lopes, Vilasbôas \& Castellanos, 2017). 
Diante disso, ressalta-se que durante a prestação dos cuidados no âmbito domiciliar, a presença do cuidador é indispensável, tal como o profissional de enfermagem, sendo o indivíduo adequadamente capacitado no auxílio do paciente em relação as suas necessidades e atividades cotidianas. Entre os principais cuidados ofertados pela enfermagem, é possível destacar a oferta de suporte nutricional em domicílio, permitindo o tratamento adequado de pacientes que apresentam doenças crônicas, além de reduzir a necessidade de internação hospitalar prolongada ou repetida, na medida em que permite a manutenção de suas necessidades nutricionais de maneira adequada e satisfatória ao seu organismo (Naves \& Tronchln, 2018).

Para a prestação de cuidados no âmbito domiciliar, torna-se fundamental que o profissional enfermeiro promova, junto aos familiares, a troca de decúbito do paciente, especialmente naqueles que apresentam mobilidade reduzida em âmbito domiciliar, visando aliviar a pressão física exercida em áreas específicas de seu corpo e garantindo a prevenção e incidência de lesões por pressão, além de possibilitar um adequado processo de recuperação por meio de estímulos físicos ao corpo durante a prestação de cuidados (Moro \& Caliri, 2016; Pozzoli \& Cecílio, 2017; Marques \& Romano-Lieber, 2014; Chayamiti \& Caliri, 2010; Borges Filho \&Mascarenhas, 2010).

Destaca-se ainda que a periodicidade entre visitas domiciliares também podem constituir um risco à saúde do paciente, na medida em que o estabelecimento de intervalos grandes de tempo entre uma visita e outra pode vir a promover um risco para indivíduos que apresentam patologias agudas ou doenças crônicas, ressaltando ainda a possibilidade de sobrecarga aplicada ao cuidador familiar que acompanha o indivíduo continuamente (Rehem $\&$ Trad, 2005).

Ressalta-se ainda outro risco a ser observado durante a realização de visitas, como a possibilidade de quedas, em especial em relação a idosos que por consequência da idade avançada e fragilidade física, tornaram-se mais dependentes de sua casa e estão submetidos a tal risco de maneira mais ampla (Marques \& Romano-Lieber, 2014; Borges, Filho, Mascarenhas \& 2010).

Além disso, em situações de transição do cuidado de enfermagem, tais como no momento da alta, destaca-se que os pacientes se encontram diante de uma situação de extrema vulnerabilidade, na qual a ocorrência de situações adversas relacionadas ao uso de medicamentos ou cuidados específicos podem ocasionar a necessidade de reinternação do indivíduo ou ainda o acionamento de serviços de urgência. Com isso, torna-se fundamental que o profissional enfermeiro possa desenvolver ações de orientação e cuidado ao paciente no 
âmbito domiciliar, visando à promoção da saúde do indivíduo e sua recuperação integral, por meio da redução de riscos adversos decorrentes do autocuidado e pela adoção de estratégias que evitem danos ao paciente e custos desnecessários ao sistema de saúde (Marques \& Romano-Lieber, 2014).

\subsection{A enfermagem e a segurança do paciente no ambiente domiciliar}

A segurança do paciente constitui tema de extrema relevância e influência que possui relação direta com a qualidade da assistência à saúde e cuidados ofertados ao paciente que está sob tratamento. Nesse sentido, destaca-se a importância da atuação dos profissionais de enfermagem na prestação de cuidados e a indispensabilidade do exercício de um papel que seja capaz de garantir a promoção de segurança ao paciente por meio de ações e atividades práticas de cuidado ofertados ao indivíduo (Pozzoli \& Cecílio, 2017; Calvalcante, Rocha, Nogueira, Avelino \& Rocha, 2015).

Desse modo, é preciso destacar que estabelecer a segurança do paciente, em especial no ambiente domiciliar, constitui tarefa que deve ser pautada pela promoção de cuidados adequados e estabelecimento de um processo comunicativo eficaz entre os cuidados e o indivíduo que recebe cuidados, permitindo o entendimento sobre as necessidades de cada ator envolvido no processo de cuidado e a minimização e extinção de potenciais riscos e danos que podem vir a ser causador por meio de uma prestação de assistência ineficiente ou que não observe atentamente os parâmetros necessários para a construção de um ambiente seguro (Rehem \& Trad, 2005; Lopes, Vilasbôas \& Castellanos, 2017).

Nessa perspectiva, observa-se como a segurança do paciente no âmbito domiciliar constitui um desafio quanto a excelência da qualidade da prestação de cuidados por parte de profissionais de enfermagem, destacando que as condições de trabalho existentes no domicílio, bem como o aprimoramento técnico e pessoal do profissional enfermeiro podem estimular a prestação da assistência e influenciar na qualidade do cuidado ofertado, sendo fundamental à garantia de um ambiente domiciliar seguro para realização do tratamento de indivíduos que permanecem a maior parte do tempo em suas respectivas residências (Silva, Alves, Sanches, Terra \& Resck, 2016).

Por fim, destaca-se ainda a indisponibilidade de estudos aprofundados acerca da referida temática, evidenciando a necessidade de desenvolvimento de novas pesquisas que envolvam a relação existente entre a enfermagem e o estabelecimento de segurança do paciente no ambiente domiciliar, visando o desenvolvimento de metodologias de cuidado, o 
aprimoramento de técnicas e competências do cuidador e a oferta de cuidados integrais e dignos, ao mesmo tempo em que se proporciona a construção de um ambiente domiciliar adequadamente seguro para o paciente sob tratamento.

\section{Considerações finais}

Diante do estudo apresentado, evidencia-se que a segurança do paciente no âmbito domiciliar constitui importante objeto de estudo, devendo ter sua relevância adequadamente compreendida por profissionais de enfermagem, cuidadores e familiares de pacientes que recebem cuidados específicos em seus respectivos domicílios, visando o desenvolvimento de práticas adequadas de cuidado que possam proporcionar a construção de um ambiente seguro a realização do tratamento necessário em cada caso, possibilitando a recuperação da saúde do indivíduo e a melhora na qualidade da assistência ofertada ao mesmo.

Conclui-se que o profissional de enfermagem possui papel fundamental no processo de estabelecimento de segurança do paciente no âmbito domiciliar, na medida em que se torna responsável pela realização das principais intervenções necessárias durante o tratamento do indivíduo em seu domicílio, bem como deve ser capaz de estabelecer um processo comunicativo eficiente entre seu paciente, familiares e demais cuidadores que auxiliam na prestação de cuidados, visando reduzir e eliminar eventuais riscos e danos, favorecendo o desenvolvimento de um ambiente que possibilite a recuperação da qualidade de vida do indivíduo e sua recuperação clínica por meio de ações que garantam integralidade, dignidade e segurança durante a prestação de assistência.

Assim, o estudo demonstra e traz para a enfermagem que os profissionais devem possuir um conjunto de competências pessoais e profissionais indispensáveis à prestação de cuidados no ambiente domiciliar, visando evitar a incidência de casos de negligência, imprudência e imperícia.

Todavia, é preciso que sejam desenvolvidos mais estudos e pesquisas na referida área, em virtude da inexistência de estudos aprofundados acerca da referida temática, fato que limitou a realização desta pesquisa, e a importância de desenvolvimento de ações mais específicas que possam garantir a melhora contínua dos cuidados prestados a pacientes no ambiente domiciliar, visando o desenvolvimento e melhora de ações de enfermagem que possam garantir maior segurança, integralidade e eficiência durante a prestação de cuidados no ambiente domiciliar. 
Diante disso, o estudo direcionou um olhar para a segurança do paciente no âmbito domiciliar no que tange a importância da adoção de práticas de enfermagem seguras. Uma das limitações do estudo foi a não realização da análise de artigos indexados em bases de dados internacionais que poderia evidenciar um panorama internacional a respeito da segurança do paciente no âmbito domiciliar.

\section{Referências}

Tase, T. H., Quadrado, E. R. S. \& Tronchin, D. M. R. (2018). Evaluation of the risk of misidentification of women in a public maternity hospital. Revista Brasileira de Enfermagem, 71(1), 120-5.

Golle, L., Ciotti, D., Herr, G. E. G., Aozone, F., Schmidt, C. R. \& Kolankiewicz, A. C. B. (2018). Culture of patient safety in hospital private. Revista de Pesquisa Cuidado é Fundamental, 10(1), 85-89.

Lemos, G. C., Azevedo, C., Bernardes, M. F. V. G., Ribeiro, H. C. T. C., Menezes, A. C. \& Mata, L. R. F. (2018). The patient safety culture in the scope of nursing: theoretical reflextion. Revista de Enfermagem do Centro-Oeste Mineiro, 8, e2600.

Institute of Medicine. (1999). To err is human: building a safer health system. Washington: National Academic Press; 1999.

Cunha, J. J. C., Santos, P. N. D., Lepinski, A. G., Santos, M. M. S., Hermann, A. P. \& Lacerda, M. R. (2014). Gerenciando a assistência domiciliar. Revista Baiana de Enfermagem, 28(2),186-95.

Dalben, L. W. (2011). Gestão em cuidado domiciliar. In: Harada, M. J. C. S. (Org.). Gestão em enfermagem: ferramenta para prática segura. São Caetano do Sul: Yendis.

World Health Organization. (2009). The International Classification for Patient Safety (ICPS) Taxonomy: more than words. Geneva; 2009. 
Brasil. (2013). Portaria n. 529, de 01 de abril de 2013. Institui o Programa Nacional de Segurança do Paciente (PNSP). [Internet]. Disponível em: http://bvsms.saude.gov.br/bvs/saudelegis/gm/2013/prt0529_01_04_2013.html.

Aguiar, R. S., Santana, D. C. \& Santana, P. C. (2015). A percepção do enfermeiro da estratégia saúde da família sobre a saúde do homem. Revista de Enfermagem do Centro-Oeste Mineiro, 5 (3), 1844-54.

Mendes, K. D. S., Silveira, R. C. C. P., Galvão, C. M. (2019). Uso de gerenciador de referências bibliográficas na seleção dos estudos primários em revisão integrativa. Texto \& Contexto Enfermagem, 28, e20170204.

Rehem, T. C. M. S. B. \& Trad, L. A. B. (2005). Assistência domiciliar em saúde: subsídios para um projeto de atenção básica brasileira. Ciência \& Saúde Coletiva, 10(1), 231-42.

Gago, E. A. \& Lopes, M. J. (2012). Cuidados domiciliares: interação do enfermeiro com a pessoa idosa/família. Acta Paulista Enfermagem, 5(1), 74-80.

Cortez, E. A., Valente, G. S. C. \& Ribeiro, B. H. M. (2011). O enfermeiro frente aos riscos ocupacionais em home-care. Revista Pesquisa Cuidado é Fundamental, 3(3), 2057-70.

Vieira, H. F., Bezerra, A. L. D., Sobreira, M. V. S., Silva, J. B. \& Feitosa, A. N. A. (2015). Assistência de enfermagem ao paciente acamado em domicílio: uma revisão sistemática. FIEP BULLETIN, 85(1).

Carvalhais, M. \& Sousa, L. (2013). Qualidade dos cuidados domiciliares em enfermagem a idosos dependentes. Saúde Sociedade São Paulo, 22(1), 160-72.

Lopes, G. V. D. O., Vilasbôas, A. L. Q. \& Castellanos, M. E. P. (2017). Atenção domiciliar na estratégia saúde da família: avaliação do grau de implantação em Camaçari (BA). Saúde Debate, 41(1), 241-54. 
Monterroso, L. E. P., Sá, L. O. \& Joaquim, N. MT. (2017). Adesão ao regime terapêutico medicamentoso e aspectos biopsicossociais dos idosos integrados em cuidados continuados domiciliários. Revista Gaúcha de Enfermagem, 38(3), e56234.

Naves, L. K. \& Tronchln, D. M. R. (2018). Nutrição enteral domiciliar: perfil dos usuários e cuidadores e os incidentes relacionados às sondas enterais. Revista Gaúcha de Enfermagem, 39(1), e2017-0175.

Moro, J. V. \& Caliri, M. H. L. (2016). Úlcera por pressão após a alta hospitalar e o cuidado em domicílio. Escola Anna Nery Revista de Enfermagem, 20(3), e20160058.

Pozzoli, S. M. L. \& Cecílio, L. C. O. (2017). Sobre o cuidar e o ser cuidado na atenção domiciliar. Saúde Debate, 41(11), 1116-29.

Marques, L. F. G. \& Romano-Lieber, N. S. (2014). Segurança do paciente no uso de medicamentos após a alta hospitalar: estudo exploratório. Saúde Sociedade São Paulo, 23(4), 1431-44.

Chayamiti, E. M. P. C. \& Caliri, M. H. L. (2010). Úlcera por pressão em pacientes sob assistência domiciliar. Acta Paulista Enfermagem, 23(1), 29-34.

Borges, O. S., Filho, L. E. N. M. \& Mascarenhas, C. H. M. (2010). Correlação entre equilíbrio e ambiente domiciliar como risco de quedas em idosos com acidente vascular encefálico. Revista Brasileira de Geriatria e Gerontologia, 13(1), 41-50.

Cavalcante, A. K. C. B., Rocha, R. C., Nogueira, L. T., Avelino, F. V. S. D. \& Rocha, S. S. (2015). Cuidado seguro ao paciente: contribuições da enfermagem. Revista Cubana de Enfermagem, 31(4).

Silva, A. T., Alves, M. G., Sanches, R. S., Terra, F. S. \& Resck, Z. M. R. (2016). Assistência de enfermagem e o enfoque da segurança do paciente no cenário brasileiro. Saúde Debate, 40(1), 292-301. 
Research, Society and Development, v. 9, n. 3, e181932700, 2020

(CC BY 4.0) | ISSN 2525-3409 | DOI: http://dx.doi.org/10.33448/rsd-v9i3.2700

Andressa da Silva Alves - 60\%

Ricardo Saraiva Aguiar - 40\% 